\title{
LUTTE CONTRE LE PALUDISME DANS LE MOYEN-OUEST DE MADAGASCAR : COMPARAISON DE L'EFFICACITÉ DE LA LAMBDA-CYHALOTHRINE ET DU DDT EN ASPERSIONS INTRA-DOMICILIAIRES. II - ÉtUde PARASITOLOGIQUE ET CLINIQUE
}

\author{
COT M.*****, BRUTUS L.****, LE GOFF G.*, RAJAONARIVELO V.* \& RAVELOSON A.**
}

Summary : COMPARISON OF LAMBDA-CYHALOTHRIN AND DDT HOUSESPRAYING FOR MALARIA CONTROL ON THE WESTERN SLOPES OF

Madagascar highlands. II - Parasitological and CliniCal StUdy

For malaria vector control in Madagascar, $10 \mathrm{WP}$ (lambdacyhalothrin $10 \%$ wettable powder) was compared with DDT $75 \%$ WP for house-spraying, from November 1997 to September 1998 This study was implemented at the fringe of the malaria epidemic zone, in villages on western slopes of the central highlands, outside the area covered for the past five years by routine DDT housespraying (OPID). Four types of treatment were compared in different areas: 1) DDT $2 \mathrm{~g} \mathrm{ai} / \mathrm{m}^{2}$ and 2) lambda-cyhalothrin $30 \mathrm{mg} \mathrm{ai} / \mathrm{m}^{2}$ in previously unsprayed villages, 3) no intervention (controll);

4) yearly DDT spraying (OPID fifth cycle).

To investigate the malariological impact of spraying, cross-sectional surveys of the village populations were performed in each study area at intervals of two months, before and after spraying. In the newly sprayed areas, from December to June, malaria indices decreased by $62 \%$ in the $\mathrm{ICON}^{\circledR}$ area and $44 \%$ in the DDT area, whereas in the unsprayed village malaria increased by $32 \%$ during the same season. There was a similar decrease in the number of gametocyte carriers in the newly sprayed areas. Active malaria case detection among febrile individuals was performed fortnightly in each village outside the OPID area. Results showed decreased malaria incidence from February (two months post-sprayingl in the sprayed villages, despite the rainy season, whereas in the unsprayed area the decline occurred only after the main transmission season.

This study demonstrated that, parasitologically as well as entomologically, house-spraying with residual insecticide (DDT or ICON ${ }^{\circledR} \mid$ was an effective method for controlling malaria on the western fringes of the Madagascar highlands epidemic zone. Both products were effective, but ICON ${ }^{\circledR}$ had slightly better impact than DDT, i.e. more reduction of malaria indices and of vector longevity, less irritancy of mosquitoes. For best results in this area of transition between stable and unstable malaria, we recommend earlier annual spraying (as soon as November) and extension of the OPID barrier towards western and northern slopes of the Plateau.

KEY WORDS : malaria control, controlled trial, malaria attacks, house spraying, Madagascar, DDT, lambda-cyhalothrin

MOTS CLÉS : lutte antivectorielle, essai contrôlé, paludisme, accès palustres, aspersions intra-domiciliaires, Madagascar, DDT, lambda-cyhalothrine.

\footnotetext{
* Institut de Recherche pour le Développement (IRD), Programme Paludisme, BP 434, Antananarivo, Madagascar.

** Direction de la Lutte contre les Maladies Transmissibles (DLMT), Ministère de la santé, Antananarivo, Madagascar.

*** INSERM, unité 149, Paris, France.
}

Correspondance : M. Cot, IRD/INSERM U 149, Service de gynécologie-obstétrique, hôpital Tenon, 4, rue de la Chine, F-75020, Paris.

\section{Résumé :}

Entre novembre 1997 et septembre 1998, un essai de lutte antivectorielle portant sur l'efficacité et l'acceptabilité de I'ICON ${ }^{\circledR} 10$ WP llambda-cyhalothrine en poudre mouillable à $10 \%$, comparé à du DDT $75 \%$ WP en aspersions intra-domiciliaires, a été réalisé sur la marge ouest de la zone de paludisme instable des plateaux centraux de Madagascar, à l'extérieur de la région couverte depuis cinq ans par les opérations de pulvérisation de DDT (OPID). Quatre zones d'étude ont été définies: deux zones traitées, l'une

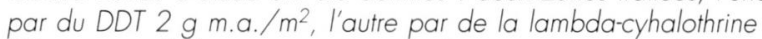
$30 \mathrm{mg}$ m.a. $/ \mathrm{m}^{2}$, une zone témoin où aucun traitement n'a été appliqué et une zone incluse dans le programme OPID $15^{\text {ème }}$ cycle annuel de pulvérisations).

Des enquêtes transversales, cliniques et parasitologiques, ont été réalisées avant et après les aspersions à intervalles de deux mois sur l'ensemble de la population des villages centrant chacune des zones. Parallèlement aux résultats entomologiques qui ont montré un effondrement des densités agressives et des taux d'infection du principal vecteur, Anopheles funestus, les indices plasmodiques on nettement diminué dans les deux zones pulvérisées par rapport à la zone témoin /diminutions de $62 \%$ et $44 \%$ respectivement six mois après les traitements par la lambda-cyhalothrine et le DDT, contre une augmentation de $32 \%$ dans la zone témoin). Cette diminution des indices plasmodiques dans les zones traitées s'est accompagnée d'une diminution des indices gamétocytaires représentant le potentiel infectant de la communauté. Le dépistage actif des cas de paludisme chez les sujets fébriles, réalisé deux fois par mois dans les trois villages situés hors de la zone OPID, a également mis en évidence une chute précoce des taux d'incidence dès le mois de février (soit deux mois après les pulvérisations) dans les zones pulvérisées, alors qu'elle ne survenait qu'en mai dans la zone témoin.

Sur le plan parasitologique comme sur le plan entomologique, cet essai a montré que l'aspersion intra-domiciliaire d'insecticides était une méthode de lutte efficace dans cette région de transition où le paludisme est modérément stable et où il existe un risque de recolonisation des plateaux par des vecteurs infectés. Les deux produits se sont révélés très actifs, avec toutefois un léger avantage pour la lambda-cyhalothrine (meilleure réduction de la longévité des vecteurs, moindre pouvoir irritant, effet plus marqué sur la réduction des indices plasmodiques) par rapport au DDT. Pour une efficacité maximale de la lutte anti-vectorielle sur les marges des hauts plateaux, nous recommandons des aspersions annuelles plus précoces (dès le mois de novembre) et couvrant une superficie plus vaste au niveau des basses terres. 
lambda-cyhalothrine dans le moyen-ouest malgache (région de transition entre la situation d'endémie stable des régions côtières et l'instabilité des plateaux centraux). Les aspersions intra-domiciliaires d'insecticides dans les deux zones d'intervention avaient entraîné une très forte diminution de la densité agressive du principal vecteur, Anopheles funestus, par rapport à une zone témoin non traitée.

En matière de lutte contre le paludisme, une réduction même importante de la transmission ne s'accompagne pas forcément d'une diminution de la prévalence parasitologique ni de la morbidité palustre au sein des populations humaines. Pour de nombreux chercheurs (Snow et al., 1988; Alonso et al., 1993; Stich et al., 1994; d'Alessandro et al., 1995; Premji et al., 1995; Nevill et al., 1996), les essais de lutte anti-vectorielle (généralement par moustiquaires imprégnées d'insecticides rémanents) ont indubitablement entraîné une réduction des accès palustres et de la mortalité, en particulier chez les jeunes enfants. Cependant, d'autres auteurs (Trape \& Rogier, 1996), s'appuyant sur le fait que des régions présentant des niveaux de transmission très différents sont relativement comparables sur le plan de la morbidité palustre, considèrent que les mesures de lutte entomologique ne peuvent suffisamment réduire les populations vectorielles pour que cet effet se traduise en santé publique.
Sans entrer dans ce débat, nous avons voulu vérifier que les résultats de notre essai sur les vecteurs de paludisme se répercutaient bien sur la santé des populations humaines, c'est pourquoi nous avons suivi, sur les plans parasitologique et clinique, les habitants des quatre zones d'étude (traitées par la lambda-cyhalothrine -ICON ${ }^{\circledR}$-, par le DDT, non traitée -zone-témoin-, ou soumise aux cycles normaux de l'OPID) avant et après l'application des insecticides.

\section{MATÉRIEL ET MÉTHODES}

\section{ZONE ET POPULATION D'ÉTUDE}

L a région d'étude a été présentée dans le précédent article; nous rappellerons les principaux $\checkmark$ points de cette description.

Trois zones de taille comparable (500 habitations soit environ 2900 habitants) ont été choisies dans le moyen-ouest de Madagascar, région de transition entre le paludisme stable des côtes et le paludisme instable des plateaux centraux, à la limite extérieure des pulvérisations intra-domiciliaires de DDT (OPID) pratiquées annuellement sur les hautes terres dans le cadre du programme national de lutte contre le palu-



Zone OPID soumise aux aspersions de DDT

Route

:ニニ_- Piste
Fig. 1. - Emplacement des villages des zones soumises à intervention et de la zone témoin (hors OPID). 
disme. L'intérieur de toutes les habitations de la première zone, centrée par deux villages mitoyens (Tsarafara et Ambararata), a été aspergé avec de la lambdacyhalothrine (ICON ${ }^{\circledR} 10$ WP) à la dose de $30 \mathrm{mg}$ $\mathrm{m} . \mathrm{a} . / \mathrm{m}^{2}$. La deuxième zone, située autour du village d'Ivory, a été aspergée de manière similaire avec du DDT $75 \%$ WP $2 \mathrm{~g} \mathrm{~m} . \mathrm{a} . / \mathrm{m}^{2}$. La troisième zone (autour du village d'Ambohimena) n'a été soumise à aucun traitement et a servi de témoin. Ces trois zones étaient situées à une altitude d'environ 900 mètres, dans un périmètre d'une quinzaine de kilomètres de diamètre (figure 1).

Une quatrième zone de taille comparable, située à proximité de la ville de Betafo (altitude $1250 \mathrm{~m}, 100$ kilomètres à l'ouest des trois zones précédentes) et régulièrement soumise à des aspersions de DDT depuis 1993 car située à l'intérieur de l'OPID, a également été sélectionnée et a permis de comparer les résultats obtenus dans la zone d'intervention à une zone traitée par insecticides pendant plusieurs années.

Les enquêtes entomologiques et parasitologiques préet post-aspersions ont porté sur les villages centrant chacune des zones (Tsarafara-Ambararata, Ivory, Ambohimena et Fenoarivo respectivement).

Un recensement des quatre zones a été réalisé en novembre 1997, préalablement aux aspersions d'insecticides. Il a été corrigé lors de la première enquête parasitologique en fonction des habitants réellement présents dans les villages, établissant les effectifs définitifs des populations suivies. Nous avons représenté sur le tableau I les chiffres de populations recensées dans les villages où se sont déroulées les enquêtes parasitologiques et entomologiques. Les deux agglomérations les plus importantes, correspondant aux deux zones traitées par insecticides, ont des populations du même ordre ( 807 habitants pour Ivory, pulvérisé par le DDT et 672 pour TsarafaraAmbararata, pulvérisé par l'ICON $\left.{ }^{\circledR}\right)$. Les deux autres localités sont de plus petite taille (310 pour Ambohimena, zone témoin, et 198 pour Fenoarivo, zone OPID). En dépit de ces différences de taille, on peut constater que les distributions par âges sont globalement comparables.

\section{INTERVENTIONS ET DÉROULEMENT DES ENQUÊTES}

Des enquêtes parasitologiques et cliniques, comportant un interrogatoire, une prise de température et un frottis-goutte épaisse pour chacun des habitants des quatre localités soumises à évaluation, ont eu lieu immédiatement avant les aspersions d'insecticides au début du mois de décembre 1997, puis tous les deux mois pendant une période de huit mois (soit en février, avril, juin et août 1998). Ces enquêtes transversales ont été réalisées par une équipe comportant un médecin et deux techniciens du laboratoire de l'IRD à Antananarivo, renforcée par les enquêteurs travaillant dans les villages (voir infra). Toute personne s'estimant malade a été examinée et traitée par le médecin. Les épisodes de fièvre isolée ont été considérés comme des accès palustres et traités par de la chloroquine $(25 \mathrm{mg} / \mathrm{kg}$ sur trois jours). Les prélèvements réalisés au cours de l'enquête ont été colorés sur place et examinés au microscope dès le retour à Antananarivo.

Par ailleurs, trois enquêteurs recrutés dans la région ont effectué des dépistages actifs des cas de paludisme en passant dans chacune des habitations des villages hors OPID tous les quinze jours, sauf au moment des enquêtes parasitologiques transversales. Les familles ont été interrogées sur la survenue d'épisodes de fièvre, la température a été vérifiée chez les sujets susceptibles d'être fébriles et un frottis et une goutte épaisse ont été pratiqués si elle dépassait $38^{\circ} \mathrm{C}$. Toute fièvre avérée isolée a été traitée comme un accès palustre selon le schéma décrit plus haut. Ces dépistages actifs se sont poursuivis jusqu'au mois de novembre 1998.

\section{RÉSULTATS}

\section{TAUX de PRÉSENTATION DE LA POPULATION}

T a présentation des habitants lors de chacune des enquêtes parasitologiques a été très bonne 1 puisque $96,1 \%$ de la population ont été prélevés en décembre 1997 (premier passage), 97,5\% en février $1998,96,7 \%$ en avril, $91,2 \%$ en juin et $89,6 \%$ en août.

\begin{tabular}{|c|c|c|c|c|c|c|c|c|c|c|}
\hline \multirow[b]{3}{*}{ Age } & \multirow{2}{*}{\multicolumn{2}{|c|}{$\begin{array}{c}\text { OPID } \\
\text { Fenoarivo }\end{array}$}} & \multicolumn{4}{|c|}{ ICON $^{\circledR}$} & \multirow{2}{*}{\multicolumn{2}{|c|}{$\begin{array}{l}\text { DDT } \\
\text { Ivory }\end{array}$}} & \multirow{2}{*}{\multicolumn{2}{|c|}{$\begin{array}{c}\text { Témoin } \\
\text { Ambohimena }\end{array}$}} \\
\hline & & & \multicolumn{2}{|c|}{ Tsarafara } & \multicolumn{2}{|c|}{ Ambararata } & & & & \\
\hline & nombre & $\%$ & nombre & $\%$ & nombre & $\%$ & nombre & $\%$ & nombre & $\%$ \\
\hline$<2$ & 12 & 6,1 & 32 & 8,2 & 22 & 7,8 & 66 & 8,2 & 18 & 5,8 \\
\hline 2 à 9 & 62 & 31,3 & 101 & 25,9 & 61 & 21,6 & 209 & 25,9 & 87 & 28,1 \\
\hline 10 à 14 & 26 & 13,1 & 50 & 12,8 & 34 & 12,1 & 105 & 13,0 & 43 & 13,9 \\
\hline$>15$ & 98 & 49,5 & 207 & 53,1 & 165 & 58,5 & 427 & 52,9 & 162 & 52,3 \\
\hline Total & 198 & & 390 & & 282 & & 807 & & 310 & \\
\hline
\end{tabular}

Tableau I. - Population d'étude (recensement de novembre-décembre 1997). 
Il faut noter que la population d'Ambohimena (zonetémoin) a été augmentée à partir du mois de février d'un quartier non recensé lors de la première enquête, portant l'effectif total de ce village de 310 à 358 .

\section{INDICES PLASMODIQUES}

Les trois zones "hors OPID" (ICON ${ }^{\circledR}$, DDT et témoin) ont été choisies de manière à être aussi semblables que possible sur les plans écologique, démographique et entomologique. Il est cependant apparu lors de l'enquête de décembre 1997 que les communautés faisant l'objet du suivi n'étaient pas impaludées de façon comparable avant les aspersions (tableau II).

L'indice plasmodique global (toutes espèces confondues) était de 38,6\% à Ivory (DDT) et de 33,5\% à Tsarafara/Ambararata $\left(\mathrm{ICON}^{\circledR}\right)$. Ces indices n'étaient donc pas très différents dans les deux villages-tests ayant bénéficié de pulvérisations. Il était notablement inférieur $(17,4 \%)$ dans le village-témoin (Ambohimena) $\left(\chi^{2}=43,9\right.$; deux degrés de liberté -ddl-; $\mathrm{p}<$ $\left.10^{-4}\right)$. L'impaludation était logiquement très faible (5,3\%) dans le village de Fenoarivo, ce que l'on peut attribuer à l'altitude mais surtout aux pulvérisations intradomiciliaires de DDT effectuées à quatre reprises depuis 1993 dans cette région concernée par l'OPID. On note toutefois que la répartition par classe d'âge des infections plasmodiales est globalement comparable d'un village à l'autre hors de la zone OPID avec un maximum de prévalence dans la tranche d'âge 2 à 9 ans (34\% à $65 \%$ selon les villages, tableau II). À
Fenoarivo en revanche, le taux de prévalence le plus élevé était atteint dans la tranche d'âge 10 à 14 ans $(8,3 \%)$, ce que l'on pourrait interpréter comme un retard à l'acquisition de la prémunition consécutif, soit à la baisse de la transmission entraînée par quatre années de pulvérisations intra-domiciliaires de DDT, soit à l'effet de l'altitude qui s'accompagne classiquement d'une transmission faible.

Après les aspersions, en dépit des différences systématiques entre les trois villages de la zone d'essai, on note une ascension progressive puis une stabilisation (correspondant à l'arrivée de la saison froide, peu propice à la multiplication des anophèles) des indices plasmodiques dans la zone témoin (de 17,4 \% à $23 \%$ entre décembre et juin, ce qui correspond à une augmentation de $32 \%)\left(\chi^{2}=6,2 ; 1 \mathrm{ddl} ; \mathrm{p}=0,01\right)$, alors qu'ils ont diminué régulièrement dans les deux zones traitées pour se positionner en-dessous du village témoin. Ils sont ainsi passés en six mois de 33,5\% à $12,7 \%$ dans la zone ICON $^{\circledR}$, soit une réduction de $62,4 \%\left(\chi^{2}=71,5 ; 1 \mathrm{ddl} ; \mathrm{p}<10^{-4}\right)$ et de $38,6 \%$ à $21,7 \%$ soit une réduction de $43,8 \%$ pour la zone DDT $\left(\chi^{2}=50,4 ; 1 \mathrm{ddl} ; \mathrm{p}<10^{-4}\right)$ (Fig. 2).

Les risques relatifs, quantifiant pour chaque enquête les risques d'infection plasmodiale par rapport à la situation initiale de décembre (tableau III), ont suivi la même évolution, avec une augmentation dans la zone-témoin après les pulvérisations et une diminution de la moitié (DDT : 0,56) à deux tiers $\left(\mathrm{ICON}^{\circledR}: 0,38\right)$ pour le risque d'impaludation au mois de juin par rapport à la période pré-intervention.

\begin{tabular}{|c|c|c|c|c|c|c|c|c|c|c|}
\hline \multirow[b]{3}{*}{ Age } & \multirow{2}{*}{\multicolumn{2}{|c|}{$\begin{array}{c}\text { OPID } \\
\text { Fenoarivo }\end{array}$}} & \multicolumn{4}{|c|}{ ICON $^{\circledR}$} & \multirow{2}{*}{\multicolumn{2}{|c|}{$\begin{array}{l}\text { DDT } \\
\text { Ivory }\end{array}$}} & \multirow{2}{*}{\multicolumn{2}{|c|}{$\begin{array}{c}\text { Témoin } \\
\text { Ambohimena }\end{array}$}} \\
\hline & & & \multicolumn{2}{|c|}{ Tsarafara } & \multicolumn{2}{|c|}{ Ambararata } & & & & \\
\hline & effectif & IP \% & effectif & IP \% & effectif & IP \% & effectif & IP \% & effectif & $\%$ \\
\hline$<2$ & 10 & 0 & 32 & 31,3 & 19 & 10,5 & 60 & 36,7 & 17 & 29,4 \\
\hline 2 à 9 & 61 & 4,9 & 101 & 58,4 & 60 & 41,7 & 205 & 64,9 & 87 & 34,5 \\
\hline 10 à 14 & 24 & 8,3 & 50 & 54 & 33 & 21,2 & 104 & 43,3 & 42 & 16,7 \\
\hline$>15$ & 95 & 5,3 & 204 & 30,4 & 151 & 17,2 & 401 & 24,2 & 153 & 6,5 \\
\hline Total & 190 & 5,3 & 387 & 40,8 & 263 & 22,8 & 770 & 38,6 & 299 & 17,4 \\
\hline
\end{tabular}

Tableau II. - Indices plasmatiques (IP) dans les quatre villages de l'étude avant les aspersions d'insecticides (décembre 1997).

\begin{tabular}{lcccc}
\hline & $\begin{array}{c}\text { Risque relatif (IC) } \\
\text { Février }\end{array}$ & $\begin{array}{c}\text { Risque relatif (IC) } \\
\text { Avril }\end{array}$ & $\begin{array}{c}\text { Risque relatif (IC) } \\
\text { Juin }\end{array}$ & $\begin{array}{c}\text { Risque relatif (IC) } \\
\text { Août }\end{array}$ \\
\hline Témoin & 1,39 & 1,47 & 1,32 & 1,01 \\
& $(1,02-1,90)$ & $(1,08-1,99)$ & $(0,97-1,81)$ & $(0,72-1,41)$ \\
New DDT & 0,83 & 0,81 & 0,56 & 0,46 \\
ICON $^{\circledR}$ & $(0,72-0,95)$ & $(0,70-0,93)$ & $(0,48-0,66)$ & $(0,38-0,55)$ \\
& 0,81 & 0,57 & 0,38 & 0,40 \\
\hline
\end{tabular}

Tableau III. - Risques relatifs d'impaludation dans les trois villages hors OPID après pulvérisations d'insecticides (classe de référence : enquête de décembre 1997 avant pulvérisations : $\mathrm{RR}=1$ ). 


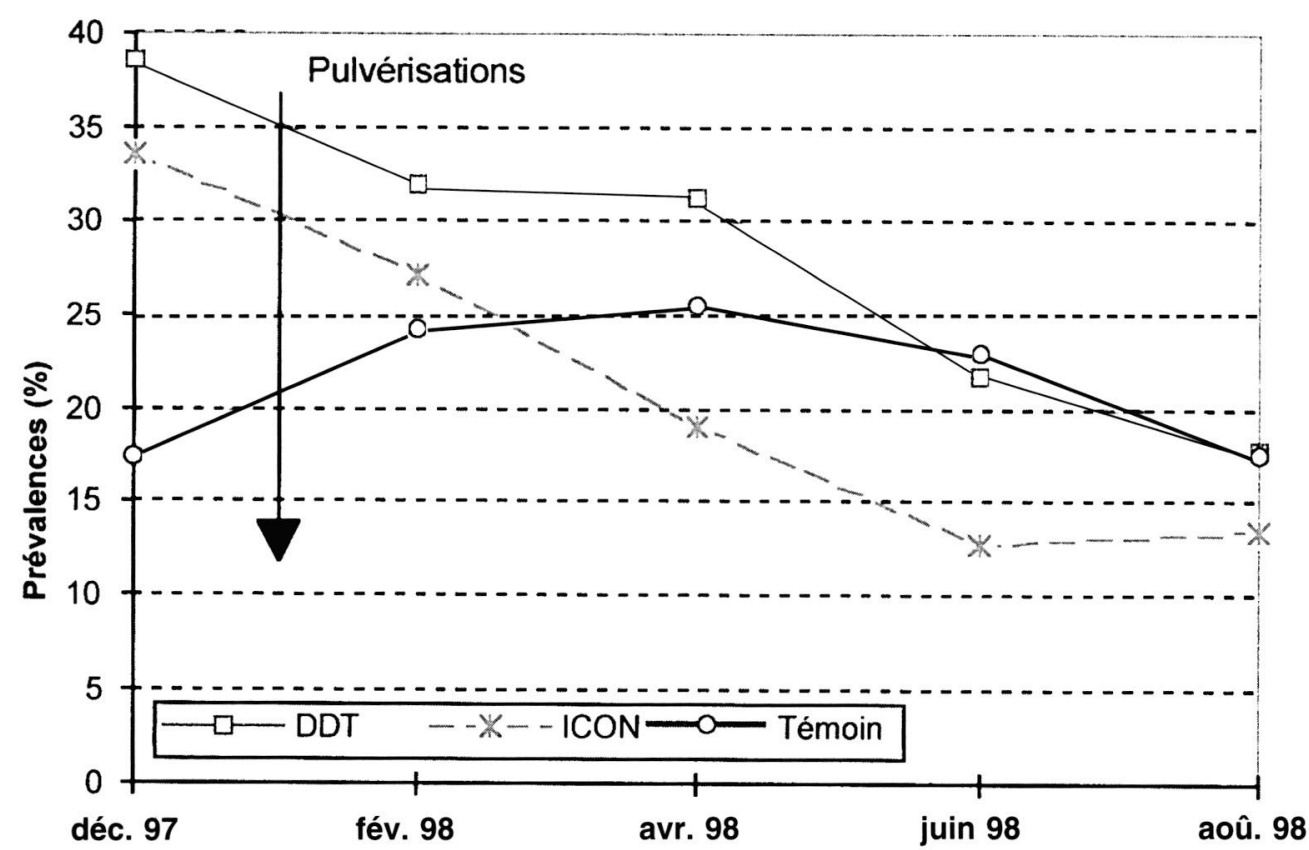

Fig. 2. - Évolution des indices plasmodiques dans les trois villages hors OPID, avant et après les pulvérisations.

Les résultats de l'enquête d'août montrent que les indices plasmodiques ont diminué dans le village témoin hors OPID (de $23 \%$ en juin à $17,5 \%$ ), ce qui correspond à l'effondrement des densités agressives des vecteurs constatée dans cette zone (Brutus et al., même numéro). Ils se sont rapprochés dans les deux villages DDT et $\operatorname{ICON}^{\circledR}(17,8 \%$ et $13,5 \%$ respectivement $)$ (figure 2). On peut attribuer cette convergence des indices des trois zones hors OPID vers des niveaux très proches à l'absence de transmission pendant toute la saison froide.

En ce qui concerne le risque relatif d'impaludation, on constate qu'il est revenu à son niveau de départ $(1,01)$ en août lorsque la transmission s'est effondrée dans la zone témoin, alors que dans les zones traitées il a continué à diminuer par rapport au mois de juin dans la zone DDT $(0,46)$ et s'est stabilisé dans la zone ICON $^{\circledR}(0,40)$ (tableau III).

Dans le village de Fenoarivo enfin, les indices plasmodiques sont restés constamment faibles, indépendamment des traitements insecticides effectués, ce qui traduit vraisemblablement l'effet cumulé des pulvérisations de DDT de l'OPID.

\section{INDICES GAMÉTOCYTAIRES À P. FALCIPARUM}

Les indices gamétocytaires sont des indicateurs importants car ils traduisent le potentiel infectant que présente la communauté humaine. On constate qu'ils ont augmenté à partir de février dans le village témoin $(2,3$ à 6,3), en suivant le premier pic de transmission traditionnellement observé en janvier, puis se sont stabilisés en juin autour de 5 lorsque la transmission était faible et ont diminué nettement en août où ils sont revenus à une valeur proche du premier passage préaspersion $(2,7)$. Dans les deux zones traitées, ils ont diminué nettement jusqu'en juin, cette diminution semblant un peu plus marquée pour les villages aspergés par l'ICON ${ }^{\circledR}$ que par le DDT (moins de 1 contre 2,6) (tableau IV). À partir d'août, les indices sont légèrement remontés dans la zone ICON ${ }^{\circledR}$, tout en restant en-deçà des valeurs relevées dans la zone traitée par le DDT. Dans la zone OPID traitée depuis plusieurs années par le DDT, ces indices sont logiquement restés en-dessous de 1,5 pendant toute la période d'observation.

\section{Dépistage ACTIF DES CAS}

Dix-sept passages ont été effectués entre janvier et novembre 1998; 589 sujets présentant une hyperthermie ont été prélevés et 138, soit $23 \%$ d'entre eux, ont été trouvés porteurs de plasmodies (toutes espèces confondues, Plasmodium falciparum représentant entre $82 \%$ et $87 \%$ des parasites selon les passages). Les taux moyens d'incidence de parasitémies fébriles par période de quinze jours, calculés sur l'ensemble de la période d'observation, étaient de 1,2\% dans la zone non traitée d'Ambohimena (valeurs extrêmes selon les passages : 0 et 3,5\%), de $0,6 \%$ dans la zone de Tsa- 


\begin{tabular}{|c|c|c|c|c|c|c|c|c|c|c|}
\hline \multirow[b]{3}{*}{ Age } & \multirow{2}{*}{\multicolumn{2}{|c|}{$\begin{array}{c}\text { OPID } \\
\text { Fenoarivo }\end{array}$}} & \multicolumn{4}{|c|}{$\operatorname{ICON}^{\circledR}$} & \multirow{2}{*}{\multicolumn{2}{|c|}{$\begin{array}{c}\text { New DDT } \\
\text { Ivory }\end{array}$}} & \multirow{2}{*}{\multicolumn{2}{|c|}{$\begin{array}{c}\text { Témoin } \\
\text { Ambohimena }\end{array}$}} \\
\hline & & & \multicolumn{2}{|c|}{ Tsarafara } & \multicolumn{2}{|c|}{ Ambararata } & & & & \\
\hline & $\mathrm{n}$ & $\%$ & $\mathrm{n}$ & $\%$ & $\mathrm{n}$ & $\%$ & $\mathrm{n}$ & $\%$ & $\mathrm{n}$ & $\%$ \\
\hline Décembre & 2 & 1,1 & 23 & 5,9 & 7 & 2,7 & 61 & 7,9 & 7 & 2,3 \\
\hline Février & 3 & 1,5 & 21 & 5,9 & 6 & 2,4 & 63 & 7,9 & 21 & 6,3 \\
\hline Avril & 2 & 1,0 & 13 & 3,5 & 4 & 1,7 & 39 & 5,1 & 19 & 5,4 \\
\hline Juin & 1 & 0,5 & 3 & 0,9 & 1 & 0,5 & 19 & 2,6 & 16 & 4,7 \\
\hline Août & 1 & 0,5 & 5 & 1,5 & 3 & 1,4 & 18 & 2,6 & 9 & 2,7 \\
\hline
\end{tabular}

n : nombre de porteurs de gamétocytes de P. falciparum.

Tableau IV. - Indices gamétocytaires dans les quatre villages de l'étude.

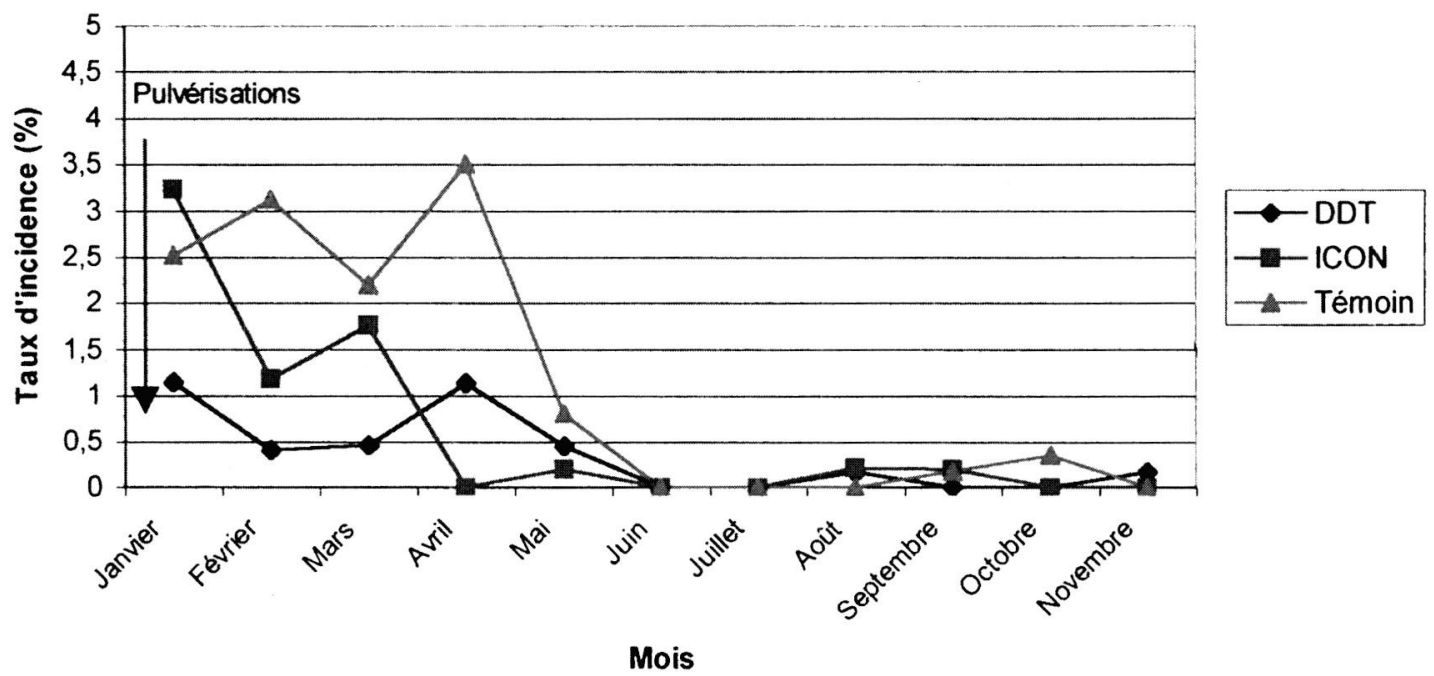

Fig. 3. - Taux d'incidence des parasitémies fébriles après pulvérisations dans les trois villages hors OPID (dépistage actif bimensuel).

rafara-Ambararata traitée par l' ICON ${ }^{\circledR}$ (valeurs extrêmes 0 et 3,2 \%) et de 0,3\% dans la zone d'Ivory traitée par le DDT (valeurs extrêmes 0 et $1,2 \%)\left(\chi^{2}\right.$ global $=$ 36,4; $\left.2 \mathrm{ddl} ; \mathrm{p}<10^{-4}\right)$. On observe (figure 3 ) que les taux d'incidence ont diminué rapidement dans les deux localités traitées dès le mois de février, peut-être plus nettement dans la zone traitée par le DDT que dans la zone traitée par l'ICON ${ }^{\circledR}$, alors que cette décroissance n'est survenue qu'au mois de mai dans la zone témoin. À partir de juin (période de très faible transmission en raison des basses températures) et jusqu'au mois de novembre, les taux sont restés voisins de 0 , confirmant les résultats des enquêtes entomologiques (Brutus et al., même numéro), dans les trois groupes de villages.

\section{DISCUSSION ET CONCLUSION}

L es résultats des enquêtes parasitologiques et cliniques effectuées avant et après les aspersions d'insecticides dans le moyen-ouest de Madagascar confirment les observations entomologiques, puisqu'ils ont montré une nette réduction des indices plasmodiques dans les deux zones pulvérisées par rapport à la zone témoin. Ces indices ont diminué de $62 \%$ et $44 \%$ respectivement six mois après les traitements par la lambda-cyhalothrine et le DDT alors qu'ils ont augmenté de $32 \%$ dans la zone témoin. Par ailleurs, la diminution des indices plasmodiques dans les zones traitées s'est accompagnée d'une diminution des indices gamétocytaires.

Le dépistage actif des cas de paludisme chez les sujets fébriles, réalisé deux fois par mois dans les trois villages situés hors de la zone OPID, a également mis en évidence une chute précoce des taux d'incidence dès le mois de février (soit deux mois après les pulvérisations) dans les zones pulvérisées, alors qu'elle ne survenait qu'en mai, au moment de la baisse de transmission accompagnant la saison froide, dans la zone témoin. Si l'on admet un décalage d'environ un mois entre la réduction de la population anophélienne et sa traduction sur les indices plasmodiques humains, cette diminution rapide de l'incidence suggère une efficacité des pulvérisations non seulement sur le pic de 
transmission classiquement observé au mois de mars, mais également, en ce qui concerne notre région d'étude, sur le premier pic détecté dès le mois de janvier (Rajaonarivelo, communication personnelle).

Plusieurs études ont comparé, au Kenya, en Tanzanie et au Sénégal, la morbidité et la mortalité des populations dans des zones où la transmission du paludisme était très hétérogène (Snow et al., 1994; Mbogo et al., 1995; Snow et al., 1995; Trape \& Rogier, 1996). Ces travaux ont mis en évidence des résultats étonnamment proches sur les populations humaines, quel que soit le niveau de transmission observé (qui pouvait varier, selon les régions étudiées, d'une à plusieurs milliers de piqûres infectantes par homme et par an). Certains des auteurs (Snow et al., 1995; Trape et Rogier, 1996) ont donc postulé que seules des réductions massives de la transmission pourraient se traduire par des effets mesurables sur la santé publique, en émettant de sérieux doutes quant à la possibilité de maintenir ces effets à moyen terme.

Dans la région où s'est déroulée l'étude, le niveau de transmission dans la zone témoin non pulvérisée (Ambohimena) était de l'ordre de six piqûres potentiellement infectantes par homme et par an et dans les zones traitées il était trop faible pour être mis en évidence sur huit mois d'observation. Ces paramètres sont proches de ceux présentés dans une enquête récente menée au Mozambique (Thompson et al., 1997), où les auteurs constataient, à la différence des travaux précédemment cités, une bonne adéquation entre morbidité et transmission entre plusieurs districts d'une zone suburbaine, qu'ils attribuaient en partie au faible niveau général de la transmission entomologique dans cette région.

La comparaison de la zone traitée lors de l'essai en 1997 uniquement et de la zone située à l'intérieur du périmètre OPID (Fenoarivo), d'où le vecteur principal a presque disparu, confirme ces résultats encourageants et permet de penser qu'à long terme, l'effet des pulvérisations non seulement se maintient mais s'amplifie en diminuant très fortement le risque d'une nouvelle épidémie sur les marges des plateaux malgaches. Par ailleurs, nos résultats sont cohérents avec les études du même type qui ont été réalisées ces dernières années sur les continents américain et africain. Charlwood et al. (1995) avaient ainsi montré au Brésil une réduction (de $28 \%$ à $76 \%$ selon les régions) des cas de paludisme diagnostiqués dans les dispensaires de districts après aspersions intra-domiciliaires de lambdacyhalothrine, alors que Kroeger et al. (1995) avaient mis en évidence, sur un très vaste territoire regroupant des régions d'Equateur, du Pérou et de Colombie, une diminution de 30 à $40 \%$ des diagnostics de paludisme effectués par interrogatoire après la pose de moustiquaires imprégnées de lambda-cyhalothrine. En Afrique, Mnzava et al. (1993) ont montré en Tanzanie une réduction significative des prévalences de parasitémies et d'accès fébriles chez les enfants, plus marquée dans des villages traités par lambda-cyhalothrine en aspersions intra-domiciliaires que dans des villages traités par DDT. Enfin, Curtis et al. (1998) ont comparé, également en Tanzanie, deux méthodes de lutte anti-vectorielle par la lambda-cyhalothrine (aspersions intra-domiciliaires et moustiquaires imprégnées) et ont trouvé dans les deux cas une réduction de la probabilité de réinfection des enfants de $54 \%$ à $62 \%$.

Dans le moyen-ouest de Madagascar, les aspersions intra-domiciliaires que nous avons réalisées hors de la zone OPID ont montré leur efficacité, tant sur le plan entomologique que parasitologique. Les deux produits se sont révélés très actifs, avec toutefois un léger avantage pour la lambda-cyhalothrine (meilleure réduction de la longévité des vecteurs, moindre pouvoir irritant, effet plus marqué sur la réduction des indices plasmodiques) par rapport au DDT. Il semble néanmoins que le critère déterminant à prendre en considération pour le choix de l'un ou l'autre de ces insecticides soit leur accumulation dans l'environnement et leurs possibles effets sur la santé humaine. Le DDT fait l'objet d'accusations persistantes (Bouwman et al., 1990; Waliszewski et al., 1996; Wolff et al., 1993) qui le font abandonner par un nombre croissant de pays (Curtis, 1994).

Dans cette région de Madagascar, l'aspersion intradomiciliaire nous paraît, en dépit de son coût plus élevé, préférable à l'utilisation de moustiquaires imprégnées du fait du comportement endophile du vecteur principal, Anopheles funestus, et de l'excellente acceptabilité de cette méthode qui permet d'assurer une couverture très satisfaisante des populations à risque. Nous considérons enfin qu'en raison de la bonne rémanence des insecticides utilisés et de la précocité de la période de transmission dans cette région (Brutus et al., même numéro), la date des pulvérisations annuelles devrait être avancée au mois de novembre (au lieu de décembre, comme c'est actuellement le cas). Ces mesures de lutte anti-vectorielle devraient en outre couvrir une superficie plus large dans les zones de transition basses-terres/plateaux centraux de manière à prévenir plus efficacement les risques de réinfestation par les populations anophéliennes et donc d'épidémie sur les plateaux.

\section{RÉFÉRENCES}

Alonso P.L., Lindsay S.W., Armstrong-Schellenberg J.R.M., Keita K., Gomez P., Shenton F., Hill A.G., David P.H., Fegan G., Cham K. \& Greenwood B.M. A malaria control trial using insecticide-treated bed nets and targeted chemoprophylaxis in a rural area of The Gambia, West Africa. 6 . The impact of the interventions on mortality and mor- 
bidity from malaria. Transactions of the Royal Society of tropical Medicine and Hygiene, 1993, 87, 2 Suppl., 37-44.

Bouwman H., Cooppan R.M., Reinecke A.J. \& Becker P.J. Levels of DDT and metabolites in breast milk from KwaZulu mothers after DDT application for malaria control. Bulletin of the World Health Organization, 1990, 68 (6), 761-768.

Brutus L., le Goff G., Rasoloniaina L.G., Rajaonarivelo V., Raveloson A. \& Cot M. Lutte contre le paludisme dans le moyen-ouest de Madagascar : comparaison de l'efficacité de la lambda-cyhalothrine et du DDT en aspersions intradomiciliaires. I - Étude entomologique. Parasite, 2001, 8, 297-308.

Charlwood J.D., Alecrim W.D., Fe N., Mangabeira J. \& MarTINS V.J. A field trial with lambda-cyhalothrin (ICON) for the intradomiciliary control of malaria transmitted by Anopheles darlingi Root in Rondonia, Brazil. Acta Tropica, 1995, 60, 3-13.

CuRTIS C.F. Should DDT continue to be recommended for malaria vector control? Medical and Veterinary Entomo$\log y, 1994,8,107-112$.

Curtis C.F., Maxwell C.A., Finch R.J. \& Nuunwa K.J. A comparison of use of a pyrethroid either for house spraying or for bednet treatment against malaria vectors. Tropical Medicine and International Health, 1998, 3 (8), 619-631.

D’alessandro U., Olaleye B.O., MacGuire W., Langerock P., Bennett S., Aikins M.K., Thomson M.C., Cham M.K., Cham B.A. \& GREENwOOD B.M. Mortality and morbidity from malaria in Gambian children after introduction of an impregnated bednet programme. Lancet, 1995, 345, 479-483.

Kroeger A., Mancheno M., Alarcon J. \& Pesse K. Insecticideimpregnated bed nets for malaria control: varying experiences from Ecuador, Colombia and Peru concerning acceptability and effectiveness. American Journal of Tropical Medicine and Hygiene, 1995, 53 (4), 313-323.

Mbogo C.N.M., Snow R.W., Khamala C.P.M., Kabiru E.W., Ouma J.H., Githure J.I., Marsh K. \& Beier J.C. Relationships between Plasmodium falciparum transmission by vector populations and the incidence of severe disease at nine sites on the Kenyan coast. American Journal of Tropical Medicine and Hygiene, 1995, 52, 201-206.

Mnzava A.E.P., Rwegoshora R.T., Tanner M., Msuya F.H., Curtis C.F. \& Irare S.G. The effects of house spraying with DDT or lambda-cyhalothrin against Anopheles arabiensis on measures of malarial morbidity in children in Tanzania. Acta Tropica, 1993, 54, 141-151.

Nevill C.G., Some E.S., Mung'ala V.O., Mutemi W., New L., Marsh K., Lengeler C. \& SNOW R.W. Insecticide-treated bednets reduce mortality and severe morbidity from malaria among children on the Kenyan coast. Tropical Medicine and International Health, 1996, 1 (2), 139-146.

Premji Z., Lubega P., Hamisi Y., Mchopa E., Minjas J., CheckLEY W. \& SHIFF C. Changes in malaria associated morbidity in children using insecticide treated mosquito nets in the Bagamoyo district of Coastal Tanzania. Tropical Medicine and Parasitology, 1995, 46, 147-153.

SNOw R.W., Rowan K.M., Lindsay S.W. \& GreEnwoOd B.M. A trial of bed nets (mosquito nets) as a malaria control strategy in a rural area of The Gambia, West Africa. Transactions of the Royal Society of tropical Medicine and Hygiene, 1988, 82, 212-215.

Snow R.W., Bastos De Azevedo I., Lowe B.S., Kabiru E.W., Newill C.G., Mwankusye S., Kassiga G., Marsh K., Teuscher T. Severe childhood malaria in two areas of markedly different falciparum transmission in East Africa. Acta Tropica, 1994, 57, 289-300.

SNOw R.W. \& MARSh K. Will reducing Plasmodium falciparum transmission alter malaria transmission among African children? Parasitology Today, 1995, 11, 188-190

Stich A.H.R., Maxwell C.A., Haji A.A., Haji D.M., Machano A.Y., Mussa J.K., Matteelli A., Haji H. \& Curtis C.F. Insecticide-impregnated bed nets reduce malaria transmission in rural Zanzibar. Transactions of the Royal Society of tropical Medicine and Hygiene, 1994, 88, 150-154.

Thompson R., Begtrup K., Cuamba N., Dgedge M., Mendis N., Gamage-Mendis A., Enosse S.M., Barreto J., Sinden R.E. \& Hogh B. The Matola malaria project: a temporal and spatial study of malaria transmission and disease in a suburban area of Maputo, Mozambique. American Journal of Tropical Medicine and Hygiene, 1997, 57 (5), 550-559.

Trape J.F. \& Rogier C. Combating malaria morbidity and mortality by reducing transmission. Parasitology Today, 1996 12 (6), 236-240.

Waliszewski S.M., Pardio Sedas V.T., Chantiri P.J.N., Infanzon R.R.M. \& RIVERA J. Organochlorine pesticide residues in human breast milk from tropical areas in Mexico. Bulletin of Environmental Contamination and Toxicology, 1996, 57, 22-28.

Wolff M.S., Toniolo P.G., Lee E.W., Rivera M. \& Dubin N Blood levels of organochlorine residues and risk of breast cancer. Journal of the National Cancer Institute, 1993, 8, 648-652.

Reçu le 17 février 2001 Accepté le 27 août 2001 